\title{
How body activity and sports improve cognitive performance, wellness, and act against aging in human beings
}

\section{Opinion}

Take off a body from its place, move, shake, act, induce, excite, give the push, start, are the descriptions in the etymology of the word "movere", from which the word movement derives. Every motor action, which carries out our body and which all of us consider simple and obvious, is, instead, something organic and highly refined, which influences and depends on all the apparatuses of the human body, but principally, on the locomotor apparatus, that allows the body to move and sustain itself. From the respiratory and circulatory systems, which supply oxygen and nutrients, sustained also to the nervous system that coordinates perceptions, the position in the space of the body, and the motor action? The functions related to the movement are the support of the body, the protection of the internal organs, the connection with the other apparatuses through tendons and muscles, with the function of levers, so as to allow movement. The motor practice has innumerable beneficial effects on the human organism, for example the increased blood circulation due to physical exercise, feeds better the bone tissue supplying it with calcium, favours the development of themselves bones size, strengthens articulations and increases and recovers mobility. Furthermore, it influences breathing by improving recovery capacity after an effort, increasing vital capacity yet, and enhancing respiratory mechanics. It has effects on the heart, which becomes bigger and different in shape (athletes heart becomes almost spherical). Movement increases the heart rate under stress and decreases it during rest (brachycardia). Causes the increase of the capillaries in the muscles and the decrease of the recovery time. Last but not least, it has enormous benefits on our nervous system and on our brains. A recent study shows that boys who are well trained and provide good performance in outdoor sports have a body weight that is age-appropriate and better respond to standard tests for memory and learning functions. In fact, in a study involving about 2,000 school students in LA, subjected to a 1 kilometer and a half run, and then, to some standardized tests of intelligence according to age and socio-cultural level, it was found that boys who had run better and faster, were also able to give the best results to tests of intelligence and culture.

A clear expression of the positive correlation between motor and brain activity. A group of researchers from the University of Granada, led by Dr. Luque-Casado, compared two groups of young adults during a 60-minute cognitive activity of psychomotor vigilance. The first group consisted of individuals who regularly practiced physical activity, the other group of people leading a sedentary lifestyle. The electrophysiological activity of the participants was recorded. It was observed that those who regularly practiced physical activity had a greater ability to focus their attention efficiently throughout the assigned task than individuals who had a sedentary lifestyle. The most recent studies have highlighted how exercise activates neuronal circuits and information used in the most varied areas of human life. In a research, published in the journal Stem Cells, of the Institute of Cell Biology and Neurobiology of the National Research Council (Ibcn-
Volume 9 Issue 4 - 2018

\section{Vittorio Catalano \\ Department of Clinical Neuropsychology, Wellness Psychology, Italy}

Correspondence: Vittorio Catalano, MD Psychiatry ED, Clinical Neuropsychology, Wellness Psychology, Italy, Email maverik333@yahoo.it

Received: January 21, 2018 | Published: July 05, 2018

Cnr) of Rome, Roman researchers, working on laboratory rats with neuronal and behavioral deficits, caused by the lack of the Btg1 gene, which acts as a proliferative brake of stem cells, have found that in the brain physical exercise blocks the aging process. In practice, they have noticed that, in the absence of this gene, the deficit neurogenesis and the stem cells loss process start again when a physical activity is practised, triggering a cellular super proliferation with a prolonged effect over time. More and more research confirms that exercise is not limited to strengthening muscles and preventing cardiovascular disease. It also improves mental performance and helps to prevent diseases of the brain such as Alzheimer's disease. The most evident benefits are at the level of memory, the first brain function to "lose strokes" (begins to decline from 25 years onwards), as shown in a study conducted at Columbia University and published in the PNAS journal. Compared to sedentary people, people who perform regular physical activity are better at mnemonic tests. At the Feinberg School of Medicine at Northwestern University, Dr Benloucif, Dr Zee and colleagues found that the sedentary lifestyle contributes directly to the decline in cognitive abilities and in the quality of sleep with age. Twelve men and women, aged 67 to 86, who were functionally independent, participated in a two-week study involving a 30-minute regimen of mild physical activity, 30 minutes of social interaction and a last 30 minutes of mild to moderate physical activity. The sessions started with a warm-up and mild to moderate physical activity (walking and stationary exercises on the upper and lower parts of the body). The last period of mild to moderate physical activity included fast walking, calisthenics or dance. A 10-minute recovery activity ended the 90-minute regimen. In a high-activity regime, women had to engage in 30 minutes of jogging a day (40 minutes for men), five days a week. At the end of the two-week period, all participants showed a 4 to $6 \%$ improvement in cognitive performance and a better quality of sleep (including a deeper sleep and fewer awakenings). Larry Tucker, professor of physical education, found that adults with high levels of physical activity have telomers with a nine-year biological advantage over sedentary ones and a seven-year advantage over moderately active ones (each time a cell divides, telomeres become shorter, at the end become too small to protect chromosomes and cells age and die, causing aging. 


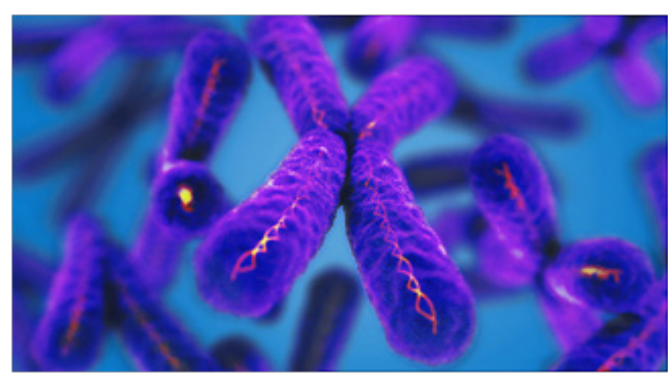

The shortening of telomeres is related to many age-related diseases, including cancer, stroke and cardiovascular disease. Whenever a biceps is contracted and released, chemicals are produced that cross the blood-brain barrier (the "curtain" that separates the brain from the bloodstream) and here stimulate the production of BDNF (brainderived neurotrophic factor). The latter contributes to "neurogenesis" (the birth of new brain cells) and to the development of new connections between one neuron and another. Although there are multiple neurotransmitters that work synergistically to regulate mood, serotonin is the main one. Its production is influenced by external factors, such as sunlight, diet and, above all, motor activity. In a study published in

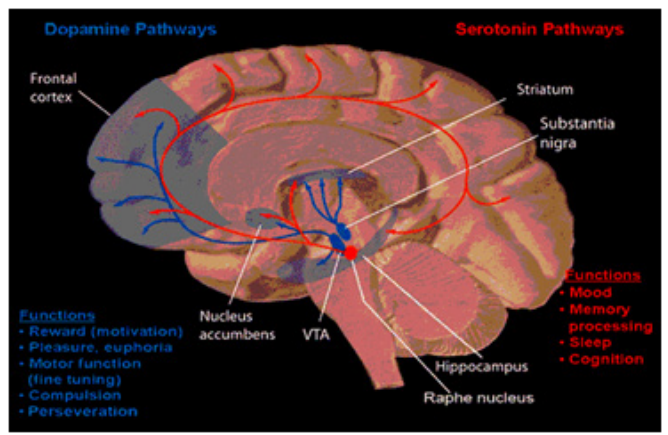

the journal of Neuropsychopharmacology, it was revealed that there are two processes, through which, the practice of sport determines a higher concentration of serotonin in the brain. First of all, motor activity increases the speed and frequency with which this hormone is released into the brain; moreover, regular physical exercise increases the secretion of tryptophan (an amino acid that is a chemical precursor of serotonin). The mechanism, however, is two-way because, as a higher quantity of serotonin improves muscle efficiency, when levels of this neurotransmitter, exceed a certain threshold, its effect becomes inhibitory rather than activating, and, can induce a sense of excessive fatigue, and make the athletic performance poorer (F. Cotel and R. Exleyb of the Univ. of Copenhagen and of Oxford). In conclusion: a correct and targeted nutrition, psychological well - being and physical activity, interacting with the positive stimulation of a balanced and healthy living environment, will allow us to lengthen and improve the quality of life and slow down aging. The knowledge and management of these correlated basic factors will also allow us to develop new targeted "interventions" for the most serious pathologies that are now common even at a young age. ${ }^{1-13}$

\section{Acknowledgements}

None.

\section{Conflict of interest}

The author declares that there is no conflict of interest.

\section{References}

1. http://www.skuola.net

2. http://www.etimo.it/

3. Studio e sport vanno d'accordo.

4. https://lamenteemeravigliosa.it/effetti-sport-cervello/

5. Ben Martynoga. How physical exercise makes your brain work better. News. 2016.

6. Redazione. II movimento e la pratica sportiva: fulcro e strumento di promozione della salute e di implementazione delle performance psicofisiche. 2017.

7. http://www.assomensana.it/Stile-di-vita-adeguato/sport-sviluppa.php

8. Intelligenza corporea: l'attività fisica é un antidepressivo, ma senza eccedere.

9. Russell Schierling. Serotonin and the Connection to Diet. 2013.

10. Ronald Klatz. Exercise-The First and Most Significant Principle in AntiAging Medicine. Total Health Magazine. 2008.

11. Jennifer Clopton. What Tiny Telomeres May Tell Us About Aging. WebMD. 2017.

12. Elizabeth Crown. Adverse Events in Hospitalized Children Are Preventable. Northwestern University; 2005.

13. Todd Hollingshead. High levels of exercise linked to nine years of less aging (at the cellular level). BYU News. 2017. 